Proceedings of the Edinburgh Mathematical Society (2004) 47, 231-249 (C)

DOI:10.1017/S0013091503000440 Printed in the United Kingdom

\title{
LOWER ESTIMATES FOR THE GROWTH OF THE FOURTH AND THE SECOND PAINLEVÉ TRANSCENDENTS
}

\author{
SHUN SHIMOMURA \\ Department of Mathematics, Keio University, 3-14-1 Hiyoshi, Kohoku-ku, \\ Yokohama 223-8522, Japan (shimomur@math.keio.ac.jp)
}

(Received 28 May 2003)

\begin{abstract}
Let $w(z)$ be an arbitrary transcendental solution of the fourth (respectively, second) Painlevé equation. Concerning the frequency of poles in $|z| \leqslant r$, it is shown that $n(r, w) \gg r^{2}$ (respectively, $n(r, w) \gg r^{3 / 2}$ ), from which the growth estimate $T(r, w) \gg r^{2}$ (respectively, $T(r, w) \gg r^{3 / 2}$ ) immediately follows.
\end{abstract}

Keywords: Painlevé transcendents; growth order; characteristic function; elliptic functions

2000 Mathematics subject classification: Primary 34M55; 34M10

\section{Introduction}

For a meromorphic function $f(z)$ in $\mathbb{C}$, denote by $n(r, f)$ the number of poles of $f(z)$ in $|z| \leqslant r$, each counted according to its multiplicity, and put

$$
\begin{gathered}
m(r, f):=\frac{1}{2 \pi} \int_{0}^{2 \pi} \log ^{+}\left|f\left(r \mathrm{e}^{\mathrm{i} \phi}\right)\right| \mathrm{d} \phi, \quad \log ^{+} x:=\max \{\log x, 0\}, \\
N(r, f):=\int_{0}^{r}(n(\rho, f)-n(0, f)) \frac{\mathrm{d} \rho}{\rho}+n(0, f) \log r .
\end{gathered}
$$

The growth of $f(z)$ is measured by the characteristic function $T(r, f):=m(r, f)+$ $N(r, f)$ or the order $\varrho(f):=\lim \sup _{r \rightarrow \infty}(\log T(r, f) / \log r$ ) (for basic facts of the value distribution theory, see, for example, $[\mathbf{5}]$ and $[\mathbf{9}])$.

All the solutions of the Painlevé equations

$$
\begin{aligned}
& w^{\prime \prime}=6 w^{2}+z, \\
& w^{\prime \prime}=2 w^{3}+z w+\alpha, \\
& w^{\prime \prime}=\frac{\left(w^{\prime}\right)^{2}}{2 w}+(3 / 2) w^{3}+4 z w^{2}+2\left(z^{2}-\alpha\right) w+\frac{\beta}{w}
\end{aligned}
$$

(where a prime denotes differentiation with respect to $z$, and $\alpha, \beta \in \mathbb{C}$ ) are meromorphic in the whole complex plane (see $[\mathbf{6}, \mathbf{1 1}, \mathbf{1 4}, \mathbf{1 5}, \mathbf{1 9}]$; see also [4]). It is an interesting and 
basic problem to estimate the growth of these solutions. For each solution $w(z)$ of $(\mathrm{I})$, (II) and (IV), the growth is evaluated as $T(r, w) \ll r^{5 / 2}, T(r, w) \ll r^{3}$ and $T(r, w) \ll$ $r^{4}$, respectively (see $[\mathbf{1 2}, \mathbf{1 6}]$; see also [4]). (We write $\chi(r) \ll \psi(r)$ or $\psi(r) \gg \chi(r)$ if $\chi(r)=O(\psi(r))$ as $r \rightarrow \infty$, and $\chi(r) \asymp \psi(r)$ if $\chi(r) \ll \psi(r) \ll \chi(r)$.) On the other hand, for the first Painlevé transcendents, i.e. the solutions of (I), the lower estimate $\varrho(w) \geqslant 5 / 2$ was given by [10], and more precise results on $T(r, w)$ have been obtained recently (see $[\mathbf{1 3}, \mathbf{1 7}])$. Furthermore, the author $[\mathbf{1 3}]$ proved that, under the condition $2 \alpha \in \mathbb{Z}$, every transcendental solution of (II) satisfies $\varrho(w) \geqslant 3 / 2$, and Steinmetz [17] obtained the following results.

(1) For most classes of transcendental solutions of (II), $\varrho(w) \geqslant 3 / 2$.

(2) For several classes of transcendental solutions of (IV), $\varrho(w) \geqslant 2$.

The purpose of this paper is to give an improvement on these results for (II) and (IV), which is stated as follows.

Theorem 1.1. Let $w(z)$ be an arbitrary transcendental solution of (IV) (respectively, (II)). Then, we have $n(r, w) \gg r^{2}$ (respectively, $\left.n(r, w) \gg r^{3 / 2}\right)$.

Remark 1.2. This theorem implies that every transcendental solution of (IV) (respectively, (II)) satisfies $T(r, w) \gg r^{2}$ (respectively, $\left.T(r, w) \gg r^{3 / 2}\right)$.

Remark 1.3. For special values of $(\alpha, \beta)$ (respectively, $\alpha$ ), equation (IV) (respectively, (II)) admits a one-parameter family of solutions such that $T(r, w) \asymp r^{2}$ (respectively, $\left.T(r, w) \asymp r^{3 / 2}\right)$ (see, for example, $\left.[\mathbf{4}, \S \S 21,25]\right)$.

In the proof, we employ a method different from those of [13] and [17]. The basic idea is, roughly speaking, to combine properties of $w(z)$ near each pole with asymptotics of Boutroux type $[\mathbf{1}, \mathbf{2}, \mathbf{8}]$; consequently, we get a chain of poles, from which our result immediately follows. Several necessary lemmas are reviewed or proved in $\S 2$. Among them, Lemmas 2.3 and 2.4 are ones concerning a kind of uniformity of a family of elliptic functions, which are essential in constructing a chain of poles. Other lemmas are mainly concerned with the behaviour of $w(z)$ of (IV) around each pole. In $\S 3$, we prove Theorem 1.1 for (IV). To do so, we treat another version of (IV) due to Boutroux $[\mathbf{1}, \mathbf{2}]$, whose solution $v(t)$ corresponding to $w(z)$ is asymptotic to an elliptic function in a suitable domain. Using this fact, we find a pair of poles of $v(t)$ that generates a chain of poles of $w(z)$. In the final section, we give an outline of the proof for (II), which is very similar to that for (IV).

Quite recently, after the submission of the original version of this paper, the author was informed that other approaches to our problem are also possible: the same result as above can be obtained through the rescaling method [18]; and the inequalities $\varrho(w) \geqslant 3 / 2$ (for (II)) and $\varrho(w) \geqslant 2$ (for (IV)) are derived by using certain estimates for logarithmic derivatives [7]. 


\section{Lemmas}

\subsection{Regular perturbation}

Denote by $B(r) \subset \mathbb{C}$ the disc defined by $|z|<r$. Let $F(x, u, \tilde{u}, \varepsilon)$ be a function analytic for $(x, u, \tilde{u}, \varepsilon) \in B\left(\xi_{0}\right) \times(\mathbb{C} \backslash\{0\}) \times \mathbb{C} \times B\left(\xi_{1}\right)$, where $\xi_{0}$ and $\xi_{1}$ are positive numbers. Consider a differential equation of the form

$$
\ddot{u}=F(x, u, \dot{u}, \varepsilon)
$$

(where the superposed dot denotes differentiation with respect to $x$ ). The following lemma is easily derived from a well-known fact on regular perturbation of differential equations (see, for example, [3, Chapter 1, Theorem 8.3]).

Lemma 2.1. Let $L, R_{0, \pm}\left(R_{0,-}<R_{0,+}\right)$ and $R_{1}$ be given positive numbers. Let $\gamma$ be an arbitrary curve starting from $x=0$ with the properties

(i) $\gamma \subset B\left(\xi_{0}\right)$;

(ii) the length of $\gamma$ does not exceed $L$.

Suppose that (2.1) with $\varepsilon=0$ admits a solution $\varphi_{0}(x)$ satisfying $\varphi_{0}(\gamma) \subset B\left(R_{0,+}\right) \backslash$ $B\left(R_{0,-}\right)$ and $\dot{\varphi}_{0}(\gamma) \subset B\left(R_{1}\right)$. Let $\varphi(\varepsilon, x)$ be the solution of (2.1) with the initial condition $\varphi(\varepsilon, 0)=\varphi_{0}(0), \dot{\varphi}(\varepsilon, 0)=\dot{\varphi}_{0}(0)$. Then, for any $\eta>0$, there exists a positive number $\varepsilon_{0}=\varepsilon_{0}\left(L, R_{0, \pm}, R_{1}, \eta\right)$ independent of $\gamma$ such that

$$
\sup _{x \in \gamma,|\varepsilon| \leqslant \varepsilon_{0}}\left|\varphi(\varepsilon, x)-\varphi_{0}(x)\right| \leqslant \eta .
$$

Remark 2.2. In the case where $F(x, u, \tilde{u}, \varepsilon)$ is analytic for $(x, u, \tilde{u}, \varepsilon) \in B\left(\xi_{0}\right) \times \mathbb{C} \times$ $\mathbb{C} \times B\left(\xi_{1}\right)$, supposing $\varphi_{0}(\gamma) \subset B\left(R_{0}\right)$ and $\dot{\varphi}_{0}(\gamma) \subset B\left(R_{1}\right)$, we have the same conclusion as above.

\subsection{A certain family of elliptic functions}

Consider a family of equations

$$
\dot{u}^{2}=P(\kappa, u):=u^{4}+4 u^{3}+4 u^{2}+\kappa u, \quad \kappa \in \mathbb{C} .
$$

Let $u=\varpi(\kappa, x)$ be an elliptic (or degenerate elliptic) function satisfying (2.3), and let $J(>1)$ be a large positive number. Naturally, $\varpi(\kappa, x)$ is not a constant function. Then we have the following lemma.

Lemma 2.3. For each $q \in \mathbb{Z}$, and for each $\kappa,|\kappa| \leqslant J$, the function $\varpi(\kappa, x)$ admits a period $\omega(\kappa)$ satisfying $c^{-} \leqslant|\omega(\kappa)| \leqslant c^{+}$and $|\arg \omega(\kappa)-(2 q-1) \pi / 4|<\pi / 4+\pi / 200$, where $c^{ \pm}=c^{ \pm}(J)$ are some positive numbers independent of $\kappa$ and $q$.

Lemma 2.4. Suppose that $|\kappa| \leqslant J$, and that $\zeta_{0}=\varpi(\kappa, 0),\left|\zeta_{0}\right|>10$. Then there exists a curve $\Gamma_{\kappa}\left(\zeta_{0}\right)$ joining 0 to $\omega(\kappa)$ with the following properties. 
(i) The length of $\Gamma_{\kappa}\left(\zeta_{0}\right)$ does not exceed $L\left(J,\left|\zeta_{0}\right|\right)$, where $L\left(J,\left|\zeta_{0}\right|\right)$ is some positive number independent of $\kappa$.

(ii) $\Lambda_{-}(J)<|\varpi(\kappa, x)|<\Lambda_{+}\left(J,\left|\zeta_{0}\right|\right)$ along $\Gamma_{\kappa}\left(\zeta_{0}\right)$, where $\Lambda_{-}(J)$ and $\Lambda_{+}\left(J,\left|\zeta_{0}\right|\right)$ are some positive numbers independent of $\kappa$.

To prove these lemmas, we examine the Riemann surface $\mathfrak{R}_{\kappa}$ of $\sqrt{P(\kappa, u)}$ and its cycles, especially in the generic case. It is easy to see that $P(\kappa, u)$ admits multiple zeros if and only if $\kappa=0$ or $\kappa=32 / 27$; then

$$
P(0, u)=u^{2}(u+2)^{2}, \quad P(32 / 27, u)=u(u+2 / 3)^{2}(u+8 / 3) .
$$

We may regard $\kappa$ as a point in the set

$$
E_{J}:=\left\{\kappa \in \mathcal{R}_{0}|| \kappa|\leqslant J,| \arg \kappa-\pi \mid \leqslant \pi\right\},
$$

where $\mathcal{R}_{0}$ denotes the universal covering of $\mathbb{C} \backslash\{0\}$.

(A) Generic case. At first we suppose that

$$
\kappa \in E^{(\mu)}:=\left\{\kappa \in \mathcal{R}_{0}|| \kappa|\geqslant \mu,| \kappa-32 / 27|\geqslant \mu,| \arg \kappa-\pi \mid \leqslant \pi\right\},
$$

where $\mu=\mu(J)$ is a small positive number which will be fixed later. Then all the zeros of $P(\kappa, u)$ are distinct, and $\varpi(\kappa, x)$ is doubly periodic. The distinct zeros are given by $u=0, u_{j}(\kappa)(j=0,1,2)$, where

(i) the $u_{j}(\kappa)$ are continuous for $\kappa \in E^{(\mu)}$; and

(ii) $u_{j}(\kappa)=(-\kappa)^{1 / 3} \mathrm{e}^{2 j \pi \mathrm{i} / 3}(1+o(1))$ as $|\kappa| \rightarrow \infty$.

To define the Riemann surface and its cycles, suppose, at first, that $-\kappa>0$, and that $|\kappa|$ is large. Let $X_{\kappa}^{l}(l=1,2)$ be two copies of $P^{1}(\mathbb{C}) \backslash\left(\Sigma_{0} \cup \Sigma_{1}\right)\left(P^{1}(\mathbb{C}):=\mathbb{C} \cup\{\infty\}\right)$ cut along the segments $\Sigma_{0}:=\left[0, u_{0}(\kappa)\right], \Sigma_{1}:=\left[u_{1}(\kappa), u_{2}(\kappa)\right]$; and denote by $\Sigma_{0}^{l,-}, \Sigma_{0}^{l,+}$ (respectively, $\Sigma_{1}^{l,-}, \Sigma_{1}^{l,+}$ ) the lower and the upper (respectively, the left and the right) edges of the cut along $\Sigma_{0}$ (respectively, $\Sigma_{1}$ ) in each $X_{\kappa}^{l}$. Gluing $\Sigma_{0}^{1, \pm}$ to $\Sigma_{0}^{2, \mp}$, and $\Sigma_{1}^{1, \pm}$ to $\Sigma_{1}^{2, \mp}$, we get, from $X_{\kappa}^{l}(l=1,2)$, the Riemann surface $\mathfrak{R}_{\kappa}$ of $\sqrt{P(\kappa, u)}$. Then, define its cycles $\gamma_{\kappa}^{1}$ and $\gamma_{\kappa}^{2}$ in the standard way.

(i) $\gamma_{\kappa}^{1}$ is a loop lying in $X_{\kappa}^{1}$, surrounding the cut $\Sigma_{0}^{1, \pm}$ in the positive sense, and not surrounding the other one $\Sigma_{1}^{1, \pm}$.

(ii) $\gamma_{\kappa}^{2}$ is a loop lying in $X_{\kappa}^{1} \cup X_{\kappa}^{2}$ such that

(a) $\gamma_{\kappa}^{2}$ surrounds the branch points 0 and $u_{1}(\kappa)$ in the positive sense, and

(b) $\gamma_{\kappa}^{2}=\gamma_{\kappa}^{2,1} \cup \gamma_{\kappa}^{2,2}$, where $\gamma_{\kappa}^{2,1}$ is a curve in $X_{\kappa}^{1}$ joining $u_{0}(\kappa) / 2 \in \Sigma_{0}^{1,+}$ to $\left(u_{1}(\kappa)+u_{2}(\kappa)\right) / 2 \in \Sigma_{1}^{1,-}$, and $\gamma_{\kappa}^{2,2}$ is a curve in $X_{\kappa}^{2}$ joining $\left(u_{1}(\kappa)+u_{2}(\kappa)\right) / 2 \in$ $\Sigma_{1}^{2,+}$ to $u_{0}(\kappa) / 2 \in \Sigma_{0}^{2,-}$. 
Furthermore, for every $\kappa \in E_{J}^{(\mu)}:=E_{J} \cap E^{(\mu)}$, we can choose the Riemann surface $\mathfrak{R}_{\kappa}$ with its cycles $\gamma_{\kappa}^{1}, \gamma_{\kappa}^{2}$ in such a way that $\mathfrak{R}_{\kappa}, \gamma_{\kappa}^{1}$ and $\gamma_{\kappa}^{2}$ are consequences of continuous deformation (with respect to $\kappa$ ) of those for special values of $\kappa$ defined above. Then $\varpi(\kappa, x)$ admits the periods

$$
\omega_{1}(\kappa):=\int_{\gamma_{\kappa}^{1}} \frac{\mathrm{d} u}{\sqrt{P(\kappa, u)}}, \quad \omega_{2}(\kappa):=\int_{\gamma_{\kappa}^{2}} \frac{\mathrm{d} u}{\sqrt{P(\kappa, u)}},
$$

which are continuous for $\kappa \in E_{J}^{(\mu)}$, and satisfy $\operatorname{Im}\left(\omega_{2}(\kappa) / \omega_{1}(\kappa)\right) \neq 0$.

(B) Degenerate and almost degenerate cases. If $\kappa=0$ (respectively, $\kappa=32 / 27$ ), then $\varpi(\kappa, x)$ is simply periodic, and admits the period

$$
\omega(0)=2 \pi \mathrm{i} \operatorname{Res}(0)=\pi \mathrm{i} \quad(\text { respectively, } \omega(32 / 27)=2 \pi \mathrm{i} \operatorname{Res}(-2 / 3)=\sqrt{3} \pi),
$$

where $\operatorname{Res}\left(u_{0}\right)$ denotes the residue of $1 / \sqrt{P(\kappa, u)}$ at the pole $u=u_{0}$. Now choose the positive number $\mu=\mu(J)$ in (2.4) so small that, for every $\kappa \in E_{J}$ satisfying $|\kappa|<\mu$ (respectively, $|\kappa-32 / 27|<\mu$ ), all zeros of $P(\kappa, u)$ remain in either of the discs $|u|<1 / 10$, $|u+2|<1 / 10$ (respectively, $|u|<1 / 10,|u+2 / 3|<1 / 10,|u+8 / 3|<1 / 10$ ), and that, for $\kappa$ with the same condition, a period of $\varpi(\kappa, x)$ given by

$$
\omega(\kappa):=\int_{|u|=1} \frac{\mathrm{d} u}{\sqrt{P(\kappa, u)}} \quad\left(\text { respectively, } \omega(\kappa):=\int_{|u+2 / 3|=1 / 3} \frac{\mathrm{d} u}{\sqrt{P(\kappa, u)}}\right)
$$

satisfies $|\omega(\kappa)-\omega(0)|<1 / 100$ (respectively, $|\omega(\kappa)-\omega(32 / 27)|<1 / 100$ ).

Proof of Lemma 2.3. We prove this lemma for $q=1$; the other cases are treated similarly. For $\kappa \in E_{J}$ satisfying $|\kappa|<\mu$ or $|\kappa-32 / 27|<\mu$, the period given by (2.6) and (2.7) satisfies $\pi / 2<|\omega(\kappa)|<2 \pi,|\arg \omega(\kappa)-\pi / 4|<\pi / 4+\pi / 200$. Next suppose that $\kappa \in E_{J}^{(\mu)}=E_{J} \cap E^{(\mu)}$. Since $E_{J}^{(\mu)}$ is compact and simply connected, the periods given by $(2.5)$ have the following properties:

$$
\begin{aligned}
M_{J}^{-} & :=\inf _{\kappa \in E_{J}^{(\mu)}} \min \left\{\left|\omega_{1}(\kappa)\right|,\left|\omega_{2}(\kappa)\right|\right\}>0, \\
M_{J}^{+} & :=\sup _{\kappa \in E_{J}^{(\mu)}} \max \left\{\left|\omega_{1}(\kappa)\right|,\left|\omega_{2}(\kappa)\right|\right\}<+\infty, \\
\eta_{J} & :=\inf _{\kappa \in E_{J}^{(\mu)}}\left|\sin \left(\arg \omega_{2}(\kappa)-\arg \omega_{1}(\kappa)\right)\right|>0 .
\end{aligned}
$$

Consider the set of lattice points $\Omega_{\kappa}=\left\{k_{1} \omega_{1}(\kappa)+k_{2} \omega_{2}(\kappa) \mid\left(k_{1}, k_{2}\right) \in \mathbb{Z}^{2}\right\}$. Since

$$
\Omega_{\kappa}^{J}:=\Omega_{\kappa} \cap\left\{x \mid 0<\operatorname{Re} x<2 M_{J}^{+}, 0<\operatorname{Im} x<2 M_{J}^{+}\right\} \neq \emptyset,
$$

we can choose $\omega(\kappa)=k_{1}^{\kappa} \omega_{1}(\kappa)+k_{2}^{\kappa} \omega_{2}(\kappa) \in \Omega_{\kappa}^{J}$ satisfying $|\arg \omega(\kappa)-\pi / 4|<\pi / 4$ and $\eta_{J} M_{J}^{-} \leqslant|\omega(\kappa)| \leqslant 2 \sqrt{2} M_{J}^{+}$. Here $\left|k_{1}^{\kappa}\right|+\left|k_{2}^{\kappa}\right| \leqslant h_{J}:=8 M_{J}^{+} /\left(\eta_{J} M_{J}^{-}\right)$for every $\kappa \in E_{J}^{(\mu)}$; indeed, even if neither of $\pm \omega_{1}(\kappa), \pm \omega_{2}(\kappa)$ is located in $\Omega_{\kappa}^{J}$, the set $\Omega_{\kappa}^{J}$ is contained in the parallelogram with sides $\left[0, \epsilon_{1} h_{J} \omega_{1}(\kappa) / 2\right],\left[0, \epsilon_{2} h_{J} \omega_{2}(\kappa) / 2\right]$ for suitably chosen signs $\epsilon_{1}, \epsilon_{2} \in\{ \pm 1\}$. Therefore, for each $\kappa$ satisfying $|\kappa| \leqslant J, 0 \leqslant \arg \kappa<2 \pi$, there exists a period $\omega(\kappa)$ with the desired property. 
Proof of Lemma 2.4. First consider the case where $\kappa \in E_{J},|\kappa|<\mu$. To the circle $|u|=1$, add the segment $\left[1,\left|\zeta_{0}\right|\right]$ (from 1 to $\left|\zeta_{0}\right|$ ), the $\operatorname{arc} u=\left|\zeta_{0}\right| \mathrm{e}^{\mathrm{i} \phi}, 0 \leqslant \phi \leqslant \arg \zeta_{0}<2 \pi$ (from $\left|\zeta_{0}\right|$ to $\zeta_{0}$ ), and their inverses. Then we obtain a new loop $\gamma_{\kappa}\left(\zeta_{0}\right)$ starting from and returning to $u=\zeta_{0}$. The function $y=\varpi(\kappa, x)$ with $\varpi(\kappa, 0)=\zeta_{0}$ satisfies

$$
x=\int_{\gamma_{\kappa}\left(\zeta_{0}\right)(y)} \frac{\mathrm{d} u}{\sqrt{P(\kappa, u)}}, \quad \text { in particular, } \omega(\kappa)=\int_{\gamma_{\kappa}\left(\zeta_{0}\right)} \frac{\mathrm{d} u}{\sqrt{P(\kappa, u)}}
$$

(cf. (2.7)), where $\gamma_{\kappa}\left(\zeta_{0}\right)(y)$ denotes the part of $\gamma_{\kappa}\left(\zeta_{0}\right)$ from the starting point $\zeta_{0}$ to $y \in \gamma_{\kappa}\left(\zeta_{0}\right)$. Let $\Gamma_{\kappa}\left(\zeta_{0}\right)$ be the image of the loop $\gamma_{\kappa}\left(\zeta_{0}\right)$ under mapping (2.8). Then, $1 \leqslant|\varpi(\kappa, x)| \leqslant\left|\zeta_{0}\right|$ along $\Gamma_{\kappa}\left(\zeta_{0}\right)$, and the length of $\Gamma_{\kappa}\left(\zeta_{0}\right)$ does not exceed

$$
\int_{\gamma_{\kappa}\left(\zeta_{0}\right)} \frac{|\mathrm{d} u|}{\mid \sqrt{P(\kappa, u)}} \leqslant(10 / 9)^{2} \int_{\gamma_{\kappa}\left(\zeta_{0}\right)}|\mathrm{d} u|<10\left|\zeta_{0}\right|,
$$

because $\left|\zeta_{0}\right|>10$, and because the zeros of $P(\kappa, u)$ remain in the disc $|u|<1 / 10$ or $|u+2|<1 / 10$ if $|\kappa|<\mu$. For $\kappa \in E_{J},|\kappa-32 / 27|<\mu$, we can choose $\Gamma_{\kappa}\left(\zeta_{0}\right)$ with the same properties. Finally, suppose that $\kappa \in E_{J}^{(\mu)}=E_{J} \cap E^{(\mu)}$, and recall the cycles $\gamma_{\kappa}^{\iota}$ $(\iota=1,2)$. Note that

$$
M_{J}^{*}:=\inf _{\kappa \in E_{J}^{(\mu)}} \min \left\{\left|u_{j}(\kappa)\right|,\left|u_{j}(\kappa)-u_{j^{\prime}}(\kappa)\right| \mid 0 \leqslant j \leqslant 2,0 \leqslant j^{\prime} \leqslant 2, j \neq j^{\prime}\right\}>0,
$$

and that $\left|u_{j}(\kappa)\right| \leqslant 2 J^{1 / 3}(j=0,1,2)$ for $|\kappa| \leqslant J$, since $J$ is large. Hence, we may suppose that

$$
\Lambda_{-}^{\prime}(J)<|u|<2 J, \quad\left|u-u_{j}(\kappa)\right|>\Lambda_{-}^{\prime}(J) \quad(j=0,1,2) \quad \text { for } u \in \gamma_{\kappa}^{\iota},
$$

where $\Lambda_{-}^{\prime}(J):=M_{J}^{*} / 4$. Since the length of $\gamma_{\kappa}^{\iota}$ is a continuous function of $\kappa \in E_{J}^{(\mu)}$, it does not exceed some positive number $L^{\prime}(J)$ independent of $\kappa$. Let $\gamma_{\kappa}=k_{1}^{\kappa} \gamma_{\kappa}^{1}+k_{2}^{\kappa} \gamma_{\kappa}^{2}$ be the composite cycle corresponding to the choice of $\omega(\kappa)$, where $\left|k_{1}^{\kappa}\right|+\left|k_{2}^{\kappa}\right| \leqslant h_{J}$ (cf. the proof of Lemma 2.3). The length of $\gamma_{\kappa}$ does not exceed $h_{J} L^{\prime}(J)$. Recall the cut planes $X_{\kappa}^{l}(l=1,2)$. For each $l$, denote by $\zeta_{0}^{(l)} \in X_{\kappa}^{l}$ the point corresponding to $\zeta_{0}$; in the case where $\zeta_{0} \in \Sigma_{0}$ (respectively, $\Sigma_{1}$ ), we regard $\zeta_{0}^{(l)}$ as a point in $\Sigma_{0}^{l,-}$ (respectively, $\Sigma_{1}^{l,-}$ ). For each $\kappa$, let $R_{\kappa}^{l}\left(\zeta_{0}\right) \subset \mathfrak{R}_{\kappa}$ be the ray starting from 0 and passing through $\zeta_{0}^{(l)}$ which is drawn in such a way that, when traversing a cut, the ray transfers to the other plane, and that $R_{\kappa}^{1}\left(\zeta_{0}\right) \cap R_{\kappa}^{2}\left(\zeta_{0}\right) \subset\left\{0, u_{j}(\kappa)(j=0,1,2)\right\}$. Then, either of $R_{\kappa}^{l}\left(\zeta_{0}\right)(l=1,2)$ intersects $\gamma_{\kappa}$. Suppose, for example, that $R_{\kappa}^{1}\left(\zeta_{0}\right) \cap \gamma_{\kappa} \ni \zeta^{\kappa}$, and take the segment $\left[\zeta_{0}, \zeta^{\kappa}\right] \subset R_{\kappa}^{1}\left(\zeta_{0}\right)$ starting from $\zeta_{0}^{(1)}$ and ending at $\zeta^{\kappa} \in \gamma_{\kappa}$. Adding $\left[\zeta_{0}, \zeta^{\kappa}\right]$ and its inverse to $\gamma_{\kappa}$, we obtain a loop $\gamma_{\kappa}\left(\zeta_{0}\right)$. Then, for each $\kappa \in E_{J}^{(\mu)}$, it has the following properties.

(i) $\gamma_{\kappa}\left(\zeta_{0}\right)$ is homotopic to $\gamma_{\kappa}$ in $\mathfrak{R}_{\kappa}$.

(ii) $\gamma_{\kappa}\left(\zeta_{0}\right)$ starts from and returns to $\zeta_{0}^{(1)} \in \mathfrak{R}_{\kappa}$.

(iii) $\Lambda_{-}^{\prime}(J)<|u|<\Lambda_{+}^{\prime}\left(J,\left|\zeta_{0}\right|\right)$ for $u \in \gamma_{\kappa}\left(\zeta_{0}\right)$, where $\Lambda_{+}^{\prime}\left(J,\left|\zeta_{0}\right|\right):=2 J+\left|\zeta_{0}\right|$. 
Consider (2.8) corresponding to $\gamma_{\kappa}\left(\zeta_{0}\right)$ of this case, and let $\Gamma_{\kappa}\left(\zeta_{0}\right)$ be the image of $\gamma_{\kappa}\left(\zeta_{0}\right)$ under it. Then, by (iii), we have $\Lambda_{-}^{\prime}(J)<|\varpi(\kappa, x)|<\Lambda_{+}^{\prime}\left(J,\left|\zeta_{0}\right|\right)$ along $\Gamma_{\kappa}\left(\zeta_{0}\right)$. Furthermore, the length of $\Gamma_{\kappa}\left(\zeta_{0}\right)$ does not exceed

$$
\int_{\gamma_{\kappa}\left(\zeta_{0}\right)} \frac{|\mathrm{d} u|}{|\sqrt{P(\kappa, u)}|}=L_{1}+2 L_{2}
$$

where

$$
L_{1}=\int_{\gamma_{\kappa}} \frac{|\mathrm{d} u|}{\mid \sqrt{P(\kappa, u)}}, \quad L_{2}=\int_{\left[\zeta_{0}, \zeta^{\kappa}\right]} \frac{|\mathrm{d} u|}{|\sqrt{P(\kappa, u)}|} .
$$

By (2.9), we have $L_{1} \leqslant h_{J} L^{\prime}(J) \Lambda_{-}^{\prime}(J)^{-2}$. If $\left[\zeta_{0}, \zeta^{\kappa}\right] \cap\left\{u|| u \mid \leqslant \Lambda_{-}^{\prime}(J)\right\}=\emptyset$, and if $\left[\zeta_{0}, \zeta^{\kappa}\right] \cap\left\{u|| u-u_{j}(\kappa) \mid \leqslant \Lambda_{-}^{\prime}(J)\right\}=\emptyset(j=0,1,2)$, then $L_{2} \leqslant\left|\zeta_{0}-\zeta^{\kappa}\right| \Lambda_{-}^{\prime}(J)^{-2} \leqslant\left(\left|\zeta_{0}\right|+\right.$ $2 J) \Lambda_{-}^{\prime}(J)^{-2}$. To treat the complementary case, suppose, for example, that $Z\left(\zeta_{0}, \kappa\right):=$ $\left[\zeta_{0}, \zeta^{\kappa}\right] \cap\left\{u|| u \mid \leqslant \Lambda_{-}^{\prime}(J)\right\} \neq \emptyset$. Observing that $\left|u_{j}(\kappa)\right| \geqslant 4 \Lambda_{-}^{\prime}(J)(j=0,1,2)$, we have

$$
\int_{Z\left(\zeta_{0}, \kappa\right)} \frac{|\mathrm{d} u|}{\mid \sqrt{P(\kappa, u)}} \leqslant \frac{2}{\Lambda_{-}^{\prime}(J)^{3 / 2}} \int_{0}^{\Lambda_{-}^{\prime}(J)} \frac{\mathrm{d} \lambda}{\lambda^{1 / 2}}=\frac{4}{\Lambda_{-}^{\prime}(J)} .
$$

Hence, $L_{2} \leqslant\left(\left|\zeta_{0}\right|+2 J\right) \Lambda_{-}^{\prime}(J)^{-2}+16 \Lambda_{-}^{\prime}(J)^{-1}$, which completes the proof.

\subsection{Behaviour of a solution of (IV) near a pole}

Let $w(z)$ be an arbitrary transcendental solution of (IV), and denote by

$$
\Psi(z):=\frac{w^{\prime}(z)^{2}}{w(z)}-w(z)^{3}-4 z w(z)^{2}-4\left(z^{2}-\alpha\right) w(z)+\frac{2 \beta}{w(z)}
$$

the auxiliary function satisfying

$$
\Psi^{\prime}(z)=-4 w(z)^{2}-8 z w(z) .
$$

Take a circle $|z|=r_{0}>10$ on which $w(z) \neq 0,-1, \infty$. Let $K$ be a positive number such that

$$
K>2 r_{0}^{-1} M\left(w ; r_{0}\right)+M\left(\Psi ; r_{0}\right)+10^{4}+4|\alpha|+2|\beta|,
$$

where $M(f ; r)=\max _{|z|=r}|f(z)|$, and put

$$
D_{K}:=\{z|| w(z)|>K| z \mid\}, \quad R(\theta):=\left\{z=r \mathrm{e}^{\mathrm{i} \theta} \mid r \geqslant r_{0}\right\} .
$$

Now we recall the following lemma (cf. [12, Proposition 5.3], and see also § 2.4).

Lemma 2.5. Suppose that $a,|a|>r_{0}$, has the following properties:

(i) $|w(a)|=K|a|$; and

(ii) for a sufficiently small positive number $\delta_{a}$,

$$
\left.\begin{array}{l}
R(\arg a) \cap\left\{z|| a\left|-\delta_{a}<\right| z|\leqslant| a \mid\right\} \subset \mathbb{C} \backslash D_{K}, \\
R(\arg a) \cap\left\{z|| a|<| z|<| a \mid+\delta_{a}\right\} \subset D_{K} ;
\end{array}\right\}
$$

and

(iii) $|\Psi(a)| \leqslant 7 K^{2}|a|^{3}$. 
Then there exist a pole $\sigma_{a}$ of $w(z)$ and the disc $\Delta\left(\sigma_{a}\right):\left|z-\sigma_{a}\right|<(11 / 10) K^{-1}\left|\sigma_{a}\right|^{-1}$ satisfying

(a) $|a|-1 / 10<\left|\sigma_{a}\right|<|a|+1 / 10$;

(b) $a \in \Delta\left(\sigma_{a}\right)$;

(c) $w(z)$ is analytic in $\Delta\left(\sigma_{a}\right) \backslash\left\{\sigma_{a}\right\}$; and

(d) $|w(z)|<K|z|$ on the circle $\partial \Delta\left(\sigma_{a}\right)$.

By the same argument as in $[\mathbf{1 2}, \S 3.2]$ (see also $\S 2.4$ ), we have the following lemma.

Lemma 2.6. Let $a^{\prime}, a^{\prime \prime} \in R\left(\arg a^{\prime}\right)=R\left(\arg a^{\prime \prime}\right)$ be points satisfying (i)-(iii) above, and let $\left(\sigma_{a^{\prime}}, \Delta\left(\sigma_{a^{\prime}}\right)\right),\left(\sigma_{a^{\prime \prime}}, \Delta\left(\sigma_{a^{\prime \prime}}\right)\right)$ be the corresponding pairs of pole and disc given by Lemma 2.5. Then one of the following cases occurs:

(i) $\Delta\left(\sigma_{a^{\prime}}\right) \cap \Delta\left(\sigma_{a^{\prime \prime}}\right)=\emptyset$; or

(ii) $\Delta\left(\sigma_{a^{\prime}}\right)=\Delta\left(\sigma_{a^{\prime \prime}}\right)$.

From these lemmas, we have the following lemma.

Lemma 2.7. Suppose that $a,|a|>r_{0}$ satisfies (i) and (ii) of Lemma 2.5. Then there exist a point $a_{*} \in R(\arg a),\left|a_{*}\right| \leqslant|a|$, a curve $C_{0}\left(a_{*}\right) \subset\left\{z|| z \mid \geqslant r_{0}\right\}$ and a pole $\sigma_{a_{*}}$ of $w(z)$ with the properties:

(a) $C_{0}\left(a_{*}\right)$ joins a point $z_{a},\left|z_{a}\right|=r_{0}$, to $a_{*}$, and its length does not exceed $\pi\left|a_{*}\right| / 2$;

(b) $|w(z)| \leqslant K|z|$ along $C_{0}\left(a_{*}\right)$, and $\left|w\left(a_{*}\right)\right|=K\left|a_{*}\right|$;

(c) $a, a_{*} \in \Delta\left(\sigma_{a_{*}}\right)$;

(d) $w(z)$ is analytic in $\Delta\left(\sigma_{a_{*}}\right) \backslash\left\{\sigma_{a_{*}}\right\}$; and

(e) $|w(z)|<K|z|$ on the circle $\partial \Delta\left(\sigma_{a_{*}}\right)$.

Proof. Draw the ray $R_{a}=R(\arg a)$. By (2.12), at the starting point $z_{a}=r_{0} \mathrm{e}^{\mathrm{i} \arg a}$, we have the inequalities $\left|w\left(z_{a}\right)\right|<K r_{0} / 2,\left|\Psi\left(z_{a}\right)\right|<K$; and hence $z_{a} \notin D_{K}$. Start from $z_{a}$ and proceed along $R_{a}$. There exists a point $a_{1}$ satisfying (i) and (ii) of Lemma 2.5 (with $a_{1}$ instead of $a$ ) and $\left[z_{a}, a_{1}\right] \subset \mathbb{C} \backslash D_{K}$. Then, by (2.11),

$$
\begin{aligned}
\left|\Psi\left(a_{1}\right)\right| & \leqslant\left|\Psi\left(z_{a}\right)\right|+4 \int_{\left[z_{a}, a_{1}\right]}\left(|w(s)|^{2}+2|s w(s)|\right)|\mathrm{d} s| \\
& \leqslant K+4\left(K^{2}\left|a_{1}\right|^{2}+2 K\left|a_{1}\right|^{2}\right)\left|a_{1}\right| \\
& \leqslant 4 K^{2}\left|a_{1}\right|^{3}\left(1+2 K^{-1}+K^{-1}\left|a_{1}\right|^{-3}\right) \\
& \leqslant 5 K^{2}\left|a_{1}\right|^{3}
\end{aligned}
$$

which implies that (iii) is also fulfilled. By Lemma 2.5, there exist a pole $\sigma_{a_{1}}$ and the disc $\Delta\left(\sigma_{a_{1}}\right) \ni a_{1}$. If $a=a_{1}$ or if $a \in \Delta\left(\sigma_{a_{1}}\right)$, then we get the point $a_{*}=$ 
$a_{1}$ and the segment $C_{0}\left(a_{*}\right)=\left[z_{a}, a_{*}\right]$ satisfying (a)-(e). If $a \neq a_{1}$, and if $a \notin$ $\Delta\left(\sigma_{a_{1}}\right)$, then we take the shorter arc $C_{1}$ of the circle $\partial \Delta\left(\sigma_{a_{1}}\right)$ from $b_{1}^{-}$to $b_{1}^{+}$, where $b_{1}^{-}, b_{1}^{+} \in \partial \Delta\left(\sigma_{a_{1}}\right) \cap\left(R_{a} \cup[0, a]\right),\left|b_{1}^{-}\right|<\left|b_{1}^{+}\right|$. Replacing the segment $\left[b_{1}^{-}, b_{1}^{+}\right] \subset R_{a}$ by $C_{1}$, we get the path $R_{a}^{(1)}=\left(R_{a} \backslash\left[b_{1}^{-}, b_{1}^{+}\right]\right) \cup C_{1}$. (When $z_{a} \in \Delta\left(\sigma_{a_{1}}\right)$, we put

$$
R_{a}^{(1)}=\left(\left(R_{a} \backslash\left[b_{1}^{-}, b_{1}^{+}\right]\right) \cup C_{1}\right) \cap\left\{z|| z \mid \geqslant r_{0}\right\},
$$

which starts from a point $z_{a}^{\prime},\left|z_{a}^{\prime}\right|=r_{0}$.) Let us restart from $b_{1}^{+}$and proceed along $R_{a}^{(1)}$ until we meet a point $a_{2}$ satisfying (i) and (ii) of Lemma 2.5 (with $a_{2}$ ). Denote by $R_{a}^{(1)}\left[a_{2}\right]$ the part of $R_{a}^{(1)}$ from $z_{a}$ (or $z_{a}^{\prime}$ ) to $a_{2}$. Then the length of $R_{a}^{(1)}\left[a_{2}\right]$ does not exceed $\pi\left|a_{2}\right| / 2$, and $|w(z)| \leqslant K|z| \leqslant K\left|a_{2}\right|$ on $R_{a}^{(1)}\left[a_{2}\right]$. Hence

$$
\begin{aligned}
\left|\Psi\left(a_{2}\right)\right| & \leqslant\left|\Psi\left(z_{a}\right)\right|+4 \int_{R_{a}^{(1)}\left[a_{2}\right]}\left(|w(s)|^{2}+2|s w(s)|\right)|\mathrm{d} s| \\
& \leqslant K+2\left(K^{2}\left|a_{2}\right|^{2}+2 K\left|a_{2}\right|^{2}\right) \pi\left|a_{2}\right| \\
& \leqslant 2 \pi K^{2}\left|a_{2}\right|^{3}\left(1+2 K^{-1}+K^{-1}\left|a_{2}\right|^{-3}\right) \\
& \leqslant 7 K^{2}\left|a_{2}\right|^{3}
\end{aligned}
$$

Applying Lemma 2.5, we get a pole $\sigma_{a_{2}}$ and the disc $\Delta\left(\sigma_{a_{2}}\right) \ni a_{2}$. By Lemma 2.6, $\Delta\left(\sigma_{a_{1}}\right) \cap \Delta\left(\sigma_{a_{2}}\right)=\emptyset$. If $a \notin \Delta\left(\sigma_{a_{2}}\right)$, we make the same replacement as that for $\partial \Delta\left(\sigma_{a_{1}}\right)$, and obtain a new path $R_{a}^{(2)}$, which is a modification of $R_{a}^{(1)}$. We repeat this procedure. As far as $\left|a_{j}\right|<|a|$, the area of $\Delta\left(\sigma_{a_{j}}\right)$ is not less than $\pi K^{-2}\left|\sigma_{a_{j}}\right|^{-2} \geqslant \pi K^{-2}(|a|+1)^{-2}$. By this fact and Lemmas 2.5 and 2.6, we arrive at the case of $a=a_{p}$ or of $a \in \Delta\left(\sigma_{a_{p}}\right)$ for some $p$, and get the point $a_{*}=a_{p} \in R_{a}$ and a curve $C_{0}\left(a_{*}\right)$ terminating in $a_{*}$ with length not exceeding $\pi\left|a_{*}\right| / 2$. Clearly, $a_{*}, \sigma_{a_{*}}, C_{0}\left(a_{*}\right)$ and $\Delta\left(\sigma_{a_{*}}\right)$ fulfil (a)-(e).

Lemma 2.8. Suppose that $a,|a|>r_{0}$, satisfies $|w(a)|=K|a|$. Then there exist a pole $\sigma_{a}^{\sharp}$ of $w(z)$ and the disc $\Delta\left(\sigma_{a}^{\sharp}\right)$ such that $a \in \Delta\left(\sigma_{a}^{\sharp}\right)$.

Proof. If (2.13) is fulfilled, then, by Lemma 2.7, the conclusion with $\sigma_{a}^{\sharp}=\sigma_{a_{*}}$ immediately follows. If (2.13) is not fulfilled, then there exist two possibilities:

(1) $R(\arg a) \cap\left\{z|| a\left|-\delta_{a}<\right| z|<| a \mid\right\} \subset D_{K}$; and

(2) neither (1) nor (2.13) is valid.

Note that $r_{0} \mathrm{e}^{\mathrm{i} \arg a} \in R(\arg a) \backslash\left(D_{K} \cup \partial D_{K}\right)$. In case (1), we can take the segment $\left[a^{\prime}, a\right] \subset R(\arg a), r_{0}<\left|a^{\prime}\right|<|a|$ such that $|w(z)| \geqslant K|z|$ along $\left[a^{\prime}, a\right]$, and such that (2.13) with $a^{\prime}$ (instead of $a$ ) is valid. In case (2), we can take an $\operatorname{arc} \gamma\left(a, a^{\prime \prime}\right) \subset \partial D_{K}$ joining $a$ to $a^{\prime \prime}$ such that (2.13) with $a^{\prime \prime}$ is valid. In both cases, by Lemma 2.7 , there exist a point $a_{*}^{\sharp}$, a pole $\sigma_{a}^{\sharp}:=\sigma_{a_{*}^{\sharp}}$ and the disc $\Delta\left(\sigma_{a}^{\sharp}\right)$ such that $a^{\prime} \in \Delta\left(\sigma_{a}^{\sharp}\right)$ (respectively, $a^{\prime \prime} \in \Delta\left(\sigma_{a}^{\sharp}\right)$ ). By (e) of Lemma 2.7, we have $\left[a^{\prime}, a\right] \subset \Delta\left(\sigma_{a}^{\sharp}\right)$ (respectively, $\gamma\left(a, a^{\prime \prime}\right) \subset \Delta\left(\sigma_{a}^{\sharp}\right)$ ), and hence $a \in \Delta\left(\sigma_{a}^{\sharp}\right)$, which completes the proof.

Lemma 2.9. Let $\sigma$ be an arbitrary pole of $w(z)$ such that $|\sigma|>2 r_{0}$. Then there exist a point $a^{\sigma} \in \Delta(\sigma)$ and a curve $C_{0}\left(a^{\sigma}\right) \subset\left\{z|| z \mid \geqslant r_{0}\right\}$ with the following properties: 
(i) $C_{0}\left(a^{\sigma}\right)$ joins a point $z^{\sigma},\left|z^{\sigma}\right|=r_{0}$, to $a^{\sigma}$, and the length of it does not exceed $\pi\left|a^{\sigma}\right| / 2 ;$ and

(ii) $|w(z)| \leqslant K|z|$ along $C_{0}\left(a^{\sigma}\right)$, and $\left|w\left(a^{\sigma}\right)\right|=K\left|a^{\sigma}\right|$.

Proof. Draw the ray $R(\arg \sigma)$, and take a point $a$ satisfying (2.13) and

$$
|w(z)| \geqslant K|z| \text { along }[a, \sigma] \subset R(\arg \sigma)=R(\arg a), \quad|w(a)|=K|a| .
$$

By Lemma 2.7, there exist a point $a_{*} \in R(\arg a),\left|a_{*}\right| \leqslant|a|$, a curve $C_{0}\left(a_{*}\right)$ and a pole $\sigma_{a_{*}}$ satisfying (a) and (b) of Lemma 2.7 , and

(c) $a, a_{*} \in \Delta\left(\sigma_{a_{*}}\right)$;

(d) $w(z)$ is analytic in $\Delta\left(\sigma_{a_{*}}\right) \backslash\left\{\sigma_{a_{*}}\right\}$;

(e) $|w(z)|<K|z|$ on $\partial \Delta\left(\sigma_{a_{*}}\right)$.

Put $a^{\sigma}=a_{*}$. Then, by (c) and (e) and (2.14), the pole $\sigma$ belongs to $\Delta\left(\sigma_{a_{*}}\right)$; and, by (d), $\sigma=\sigma_{a_{*}}$. In this way we have shown that $a^{\sigma} \in \Delta(\sigma)$ has the desired properties.

\subsection{Proofs of Lemmas 2.5 and 2.6}

A. As was explained in $[\mathbf{1 2}, \S 5.2]$, Lemma 2.5 (i.e. [12, Proposition 5.3]) is proved by the same argument as that used in the proof of [12, Proposition 3.1]. We sketch the proof of it; the reader can check the omitted details by consulting $[\mathbf{1 2}, \S 3]$. In addition to (2.10), recall another auxiliary function of the form

$$
\Phi(z):=\Psi(z)+\frac{4 w^{\prime}(z)}{w(z)+1},
$$

which admits the expression

$$
\begin{aligned}
\Phi(z)=E\left(z_{0}, z\right)^{-1}\left[\Phi\left(z_{0}\right)\right. & +8\left(\frac{w\left(z_{0}\right)}{\left(w\left(z_{0}\right)+1\right)^{2}}-\frac{E\left(z_{0}, z\right) w(z)}{(w(z)+1)^{2}}\right) \\
-4 \int_{\gamma\left(z_{0}, z\right)} \frac{E\left(z_{0}, s\right)}{(w(s)+1)^{2}}\left(w(s)^{2}-2 s w(s)(w(s)-1)\right. & \left.-4\left(s^{2}-\alpha\right) w(s)-2 \beta\right) \mathrm{d} s \\
& \left.+16 \int_{\gamma\left(z_{0}, z\right)} \frac{E\left(z_{0}, s\right)(w(s)-1) w(s)}{(w(s)+1)^{4}} \mathrm{~d} s\right]
\end{aligned}
$$

with

$$
E\left(z_{0}, s\right):=\exp \left(2 \int_{\gamma\left(z_{0}, s\right)} \frac{w(\zeta)-1}{(w(\zeta)+1)^{2}} \mathrm{~d} \zeta\right)
$$

(cf. $[\mathbf{1 2}, \S 5.2])$. Here $\gamma\left(z_{0}, z\right)$ denotes a path joining $z_{0}$ to $z$ on which $w(s) \neq-1$, and $\gamma\left(z_{0}, s\right) \subset \gamma\left(z_{0}, z\right)$ denotes the part from $z_{0}$ to $s$. Let $a$ be a point satisfying conditions (i)(iii) of Lemma 2.5. Then, by (i), (iii), (2.12) and (2.15), we have $|\Phi(a)| \leqslant 8 K^{2}|a|^{3}$. The following lemma corresponds to [12, Lemma 3.3]. 
Lemma 2.10. Let $\gamma(z) \subset U(a):=\left\{z|| z-\left.a\left|<2 K^{-1}\right| a\right|^{-1}\right\}$ be a path starting from $a$ and ending at $z$ with the following properties:

(i) $\|\gamma(z)\|:=\int_{\gamma(z)}|\mathrm{d} s| \leqslant 1 / 10 ;$ and

(ii) $|w(s)|$ is monotone increasing along $\gamma(z)$.

Then $|\Phi(z)|<9 K^{2}|z|^{3}$.

To prove this lemma, we first note that $|w(s)| \geqslant|w(a)|=K|a| \geqslant 10^{5}$ for $s \in \gamma(z)$. By the condition $\|\gamma(z)\| \leqslant 1 / 10$, we have $0.99|a| \leqslant|s| \leqslant 1.01|a|$ and $\left|E(a, s)^{ \pm 1}\right| \leqslant$ $\exp \left(10^{-4}\right)<1.0002$ along $\gamma(z)$. Using these inequalities and (2.16), we can derive the conclusion $|\Phi(z)|<9 K^{2}|z|^{3}$ of this lemma.

Putting $y(z)=1 / w(z)$, we write (2.15) in the form

$$
y^{\prime}(z)^{2}-\frac{4 y(z)^{2} y^{\prime}(z)}{y(z)+1}-1-4 z y(z)-4\left(z^{2}-\alpha\right) y(z)^{2}+2 \beta y(z)^{4}-y(z)^{3} \Phi(z)=0,
$$

which implies that

$$
y^{\prime}(z)= \pm(1+F(z))^{1 / 2}+\frac{2 y(z)^{2}}{y(z)+1}
$$

with

$$
F(z):=4 z y(z)+4\left(z^{2}-\alpha\right) y(z)^{2}-2 \beta y(z)^{4}+y(z)^{3} \Phi(z)+\frac{4 y(z)^{4}}{(y(z)+1)^{2}} .
$$

By [12, Lemma 3.4], we choose a steepest descent path $\gamma_{0}$ starting from $a$ and ending at $a_{*}$ in such a way that $a_{*}$ satisfies $y\left(a_{*}\right)=0$ or $a_{*} \in \partial U(a)$, and that, along $\gamma_{0}$, $\mathrm{d}|y(z)| / \mathrm{d} \lambda=-|\mathrm{d} y(z) / \mathrm{d} \lambda|$ with $\lambda:=\left\|\gamma_{0}(z)\right\|$. Here $\gamma_{0}(z)$ denotes the part of $\gamma_{0}$ from $a$ to $z$. As long as $\left\|\gamma_{0}(z)\right\| \leqslant 1 / 10$, by Lemma 2.10 , we have $|F(z)| \leqslant 2 \times 10^{-3}$, which implies that $\left|y^{\prime}(z)-1\right|<3 \times 10^{-3}$ or that $\left|y^{\prime}(z)+1\right|<3 \times 10^{-3}$. Using these facts, by the same argument as in the proof of [12, Lemma 3.5], we obtain the following.

Lemma 2.11. There exists a pole $\sigma_{a}$ of $w(z)$ satisfying $\left|\sigma_{a}-a\right| \leqslant 1.01 K^{-1}|a|^{-1}<1 / 10$ and $\left|\Phi\left(\sigma_{a}\right)\right|<9 K^{2}\left|\sigma_{a}\right|^{3}$.

Instead of [12, Lemma 3.6], we have the following.

Lemma 2.12. In the disc $\left|z-\sigma_{a}\right|<(6 / 5) K^{-1}\left|\sigma_{a}\right|^{-1}$,

$$
\begin{gathered}
|\Phi(z)|<10 K^{2}|z|^{3}, \\
\left|y^{\prime}(z)-1\right|<10^{-2} \text { or }\left|y^{\prime}(z)+1\right|<10^{-2}, \\
0.99\left|z-\sigma_{a}\right| \leqslant|y(z)| \leqslant 1.01\left|z-\sigma_{a}\right| .
\end{gathered}
$$

By Lemma 2.11, we have $|a|-1 / 10<\left|\sigma_{a}\right|<|a|+1 / 10$, implying (a) of Lemma 2.5. Observing that $\left|\sigma_{a} / a\right|<(|a|+1 / 10) /|a| \leqslant 1.01$, we have $\left|\sigma_{a}-a\right| \leqslant 1.01 K^{-1}|a|^{-1} \leqslant$ $1.01^{2} K^{-1}\left|\sigma_{a}\right|^{-1}<(11 / 10) K^{-1}\left|\sigma_{a}\right|^{-1}$, implying (b). Assertion (c) immediately follows from (2.21). Note that $\left|z / \sigma_{a}-1\right|<10^{-2}$ on $\partial \Delta\left(\sigma_{a}\right)$. Hence, by $(2.21),|y(z)| \geqslant$ $0.99 \times 1.1 K^{-1}\left|\sigma_{a}\right|^{-1} \geqslant 1.07 K^{-1}|z|^{-1}$ on $\partial \Delta\left(\sigma_{a}\right)$, which implies (d). In this way we have proved Lemma 2.5 . 
B. To prove Lemma 2.6, suppose that $\Delta\left(\sigma_{a^{\prime}}\right) \cap \Delta\left(\sigma_{a^{\prime \prime}}\right) \neq \emptyset$. Then either of the two inequalities of (2.20) is valid in $\Delta\left(\sigma_{a^{\prime}}\right) \cup \Delta\left(\sigma_{a^{\prime \prime}}\right)$; and, at the point $z_{*} \in \Delta\left(\sigma_{a^{\prime}}\right) \cap \Delta\left(\sigma_{a^{\prime \prime}}\right) \cap$ $\left[\sigma_{a^{\prime}}, \sigma_{a^{\prime \prime}}\right]$, we have, for example,

$$
\left|y\left(z_{*}\right)-\left(z_{*}-\sigma_{a^{\prime}}\right)\right| \leqslant 10^{-2}\left|z_{*}-\sigma_{a^{\prime}}\right|, \quad\left|y\left(z_{*}\right)-\left(z_{*}-\sigma_{a^{\prime \prime}}\right)\right| \leqslant 10^{-2}\left|z_{*}-\sigma_{a^{\prime \prime}}\right| .
$$

This implies

$$
\left|\sigma_{a^{\prime}}-\sigma_{a^{\prime \prime}}\right| \leqslant 10^{-2}\left(\left|\sigma_{a^{\prime \prime}}-z_{*}\right|+\left|z_{*}-\sigma_{a^{\prime}}\right|\right)=10^{-2}\left|\sigma_{a^{\prime}}-\sigma_{a^{\prime \prime}}\right|,
$$

and hence $\sigma_{a^{\prime}}=\sigma_{a^{\prime \prime}}$, from which Lemma 2.6 immediately follows.

\section{Proof of Theorem 1.1 for (IV)}

Let $w(z)$ be an arbitrary transcendental solution of (IV), and let $\Psi(z)$ be the related auxiliary function defined by (2.10). Recall the circle $|z|=r_{0}>10$ on which $w(z) \neq$ $0,-1, \infty$. In what follows, we suppose that

$$
K-1>2 r_{0}^{-1} M\left(w ; r_{0}\right)+M\left(\Psi ; r_{0}\right)+10^{4}+4|\alpha|+2|\beta|
$$

(instead of (2.12)), which means that $K$ itself also satisfies (2.12).

\subsection{Equation of Boutroux type}

Make the change of variables:

$$
t=z^{2} / 2, \quad v(t)=z^{-1} w(z) .
$$

Then $v=v(t)$ satisfies an equation of the form

$$
2 v_{t t}=\frac{v_{t}^{2}}{v}+3 v^{3}+8 v^{2}+4 v-2 t^{-1} v_{t}-2 \alpha t^{-1} v+(1 / 4) t^{-2} v+\frac{\beta t^{-2}}{2 v}
$$

(where the subscript ' $t$ ' denotes differentiation with respect to $t$ ). Multiplying both sides by $v_{t} / v$, we see that the function

$$
V(t):=\frac{v_{t}(t)^{2}}{v(t)}-v(t)^{3}-4 v(t)^{2}-4 v(t)+2 \alpha t^{-1} v(t)-(1 / 4) t^{-2} v(t)+\frac{\beta t^{-2}}{2 v(t)}
$$

satisfies the first-order equation

$$
V_{t}+2 t^{-1} V=2 t^{-1}\left(-v(t)^{3}-4 v(t)^{2}-4 v(t)+\alpha t^{-1} v(t)\right) .
$$

By this fact, we have

$$
V(t)=t_{0}^{2} V\left(t_{0}\right) t^{-2}-2 t^{-2} \int_{C\left(t_{0}, t\right)} s\left(v(s)^{3}+4 v(s)^{2}+4 v(s)-\alpha s^{-1} v(s)\right) \mathrm{d} s,
$$

where $C\left(t_{0}, t\right)$ denotes a path joining $t_{0}$ to $t$. 
Lemma 3.1. Let $\sigma$ be an arbitrary pole of $w(z)$ such that $|\sigma|>2 r_{0}$, and let $a^{\sigma}$ be the point given by Lemma 2.9. Then the corresponding point $t=b^{\sigma}=\left(a^{\sigma}\right)^{2} / 2$ satisfies $\left|b^{\sigma}-\sigma^{2} / 2\right|<2 / K,\left|v\left(b^{\sigma}\right)\right|=K$ and

$$
\left|V\left(b^{\sigma}\right)\right| \leqslant J_{0}, \quad\left|v_{t}\left(b^{\sigma}\right)^{2} / v\left(b^{\sigma}\right)-v\left(b^{\sigma}\right)^{3}-4 v\left(b^{\sigma}\right)^{2}-4 v\left(b^{\sigma}\right)\right| \leqslant J_{0},
$$

where $J_{0}=J_{0}(K)$ is some positive number independent of $\sigma$.

Proof. Let $\tilde{C}_{0}\left(b^{\sigma}\right)$ be the image of $C_{0}\left(a^{\sigma}\right)$ (cf. Lemma 2.9) under the mapping $t=$ $z^{2} / 2$, and let $b_{0},\left|b_{0}\right|=r_{0}^{2} / 2$, be the starting point of $\tilde{C}_{0}\left(b^{\sigma}\right)$. By Lemma 2.9 and (3.4) with $C\left(t_{0}, t\right)=\tilde{C}_{0}\left(b^{\sigma}\right)$, observing that $|v(t)| \leqslant K$ along $\tilde{C}_{0}\left(b^{\sigma}\right)$, we have

$$
\begin{aligned}
\left|V\left(b^{\sigma}\right)\right| & \leqslant\left|V\left(b_{0}\right)\right|+2\left|b^{\sigma}\right|^{-2}\left|\int_{\tilde{C}_{0}\left(b^{\sigma}\right)} s\left(v(s)^{3}+4 v(s)^{2}+4 v(s)-\alpha s^{-1} v(s)\right) \mathrm{d} s\right| \\
& \leqslant\left|V\left(b_{0}\right)\right|+2\left|b^{\sigma}\right|^{-2} \int_{\tilde{C}_{0}\left(b^{\sigma}\right)}\left(K^{3}+4 K^{2}+4 K+|\alpha| K\right)|s \mathrm{~d} s| \\
& \leqslant\left|V\left(b_{0}\right)\right|+\left|b^{\sigma}\right|^{-2}(2+|\alpha|) K^{3} \int_{C_{0}\left(a^{\sigma}\right)}|z|^{3}|\mathrm{~d} z| \\
& \leqslant\left|V\left(b_{0}\right)\right|+4\left|a^{\sigma}\right|^{-4}(2+|\alpha|) K^{3}\left(\pi\left|a^{\sigma}\right| / 2\right)^{4} \\
& \leqslant J_{0}^{\prime}(K):=\left|V\left(b_{0}\right)\right|+\pi^{4}(2+|\alpha|) K^{3} .
\end{aligned}
$$

Since $\left|V\left(b_{0}\right)\right|$ depends only on the initial values of $w(z)$ on the circle $|z|=r_{0}$, the constant $J_{0}^{\prime}(K)$ is independent of $\sigma$. Taking $J_{0}=J_{0}(K)=J_{0}^{\prime}(K)+(2|\alpha|+1) K+|\beta|$, we get (3.5). Since $a^{\sigma} \in \Delta(\sigma)$, we have

$$
\left|b^{\sigma}-\sigma^{2} / 2\right|=\left|\left(\sigma^{2}-\left(a^{\sigma}\right)^{2}\right) / 2\right|=\left|\sigma+a^{\sigma}\right|\left|\sigma-a^{\sigma}\right| / 2<(11 / 10)(|\sigma|+1) /(|\sigma| K)<2 / K,
$$

which completes the proof.

Lemma 3.2. Suppose that $b,|b|>r_{0}^{2}$, satisfies $|v(b)|=K$. Then there exists a pole $\tau_{b}$ of $v(t)$ such that $\left|b-\tau_{b}\right|<2 / K$.

Proof. Let $z=a$ be a point satisfying $a^{2} / 2=b,|w(a)|=K|a|$. By Lemma 2.8, there exists a pole $\sigma$ of $w(z)$ such that $a \in \Delta(\sigma)$. Then, $t=\tau_{b}=\sigma^{2} / 2$ is a pole of $v(t)$ such that $\left|b-\tau_{b}\right|=\left|a^{2}-\sigma^{2}\right| / 2=|a-\sigma||a+\sigma| / 2<(11 / 10)(|\sigma|+1) /(K|\sigma|)<2 / K$, which completes the proof.

\subsection{Construction of a pair of poles}

Consider the sectors $S_{q}:|\arg z-(2 q-1) \pi / 8|<\pi / 8+\delta(-3 \leqslant q \leqslant 4, \delta>0)$, the union of which covers the full neighbourhood of $z=\infty$. The image of $S_{q}$ under the mapping $t=z^{2} / 2$ is the sector $T_{q}:|\arg t-(2 q-1) \pi / 4|<\pi / 4+2 \delta$. Note that every non-zero pole of $w(z)$ belongs to at least one of the sectors $S_{q}(-3 \leqslant q \leqslant 4)$. 
Lemma 3.3. Let $\tau$ be an arbitrary pole of $v(t)$ satisfying $|\arg \tau-\pi / 4|<\pi / 4+\pi / 100$ and $|\tau|>\rho_{\infty}>4 r_{0}^{2}$, where $\rho_{\infty}$ is a sufficiently large positive number. Then there exists another pole $\tau^{\prime}$ of $v(t)$ such that

$$
0<\left|\tau^{\prime}-\tau\right|<c_{*}, \quad\left|\arg \left(\tau^{\prime}-\tau\right)-\pi / 4\right|<\pi / 4+\pi / 100,
$$

where $c_{*}$ is some positive number independent of $\tau$ and $\tau^{\prime}$.

Remark 3.4. This lemma is concerned with a pole of $v(t)$ in $T_{1}$, whose corresponding pole of $w(z)$ belongs to $S_{1}$. For poles in the other sectors $T_{q}(q \neq 1)$, we can show analogous facts.

Proof. Suppose that $\tau$ satisfies $|\arg \tau-\pi / 4|<\pi / 4+\pi / 100$ and $|\tau|>\rho_{\infty}$, where $\rho_{\infty}$ will be chosen afterwards. Let $\sigma=(2 \tau)^{1 / 2}$ be the corresponding pole of $w(z)$, which satisfies $|\sigma|>2 \sqrt{2} r_{0}$. By Lemmas 2.9 and 3.1 , there exists a point $b:=b^{(2 \tau)^{1 / 2}}$ satisfying

$$
|b-\tau|<2 / K, \quad|v(b)|=K, \quad\left|\kappa_{b}\right| \leqslant J_{0}=J_{0}(K),
$$

where

$$
\kappa_{b}:=v_{t}(b)^{2} / v(b)-v(b)^{3}-4 v(b)^{2}-4 v(b) .
$$

In (3.3), we put $t=b+x$. Then $u=u(b, x)=v(b+x)$ is a solution of

$$
2 \ddot{u}=\frac{\dot{u}^{2}}{u}+3 u^{3}+8 u^{2}+4 u-\frac{2 b^{-1}(\dot{u}+\alpha u)}{1+b^{-1} x}+\frac{b^{-2}(u+2 \beta / u)}{4\left(1+b^{-1} x\right)^{2}}
$$

(where the superposed dot denotes differentiation with respect to $x$ ), with the initial condition $u(b, 0)=v(b), \dot{u}(b, 0)=v_{t}(b)$. If $|b|$ is large, (3.9) is regarded as a perturbed equation of

$$
2 \ddot{u}=\frac{\dot{u}^{2}}{u}+3 u^{3}+8 u^{2}+4 u .
$$

Let $u_{0}(x)$ be a solution of (3.10) satisfying $u_{0}(0)=u(b, 0)=v(b), \dot{u}_{0}(0)=\dot{u}(b, 0)=v_{t}(b)$. Since

$$
v_{t}(t)=-z^{-2}\left(z^{-1} w(z)\right)-\left(z^{-1} w(z)\right)^{2}(1 / w(z))^{\prime}=-z^{-2} v(t)-v(t)^{2} y^{\prime}(z),
$$

by $(2.20)$, we have $\left|v_{t}(b)\right| \geqslant K^{2} / 2>0$, guaranteeing that $u_{0}(x)$ is not a constant function. This is also a solution of (2.3) with $\kappa=\kappa_{b}$ (cf. (3.8)), and we write $u_{0}(x)=\varpi\left(\kappa_{b}, x\right)$. To apply Lemma 2.1, we choose constants successively by the following process.

(i) Let $J_{0}$ be the constant of (3.7) given by Lemma 3.1. In Lemma 2.3, we put $J=J_{0}$. Then, by (3.7) and Lemma 2.3, the elliptic function $u_{0}(x)=\varpi\left(\kappa_{b}, x\right)$ admits a period $\omega\left(\kappa_{b}\right)$ such that

$$
c_{0}^{-} \leqslant\left|\omega\left(\kappa_{b}\right)\right| \leqslant c_{0}^{+}, \quad\left|\arg \omega\left(\kappa_{b}\right)-\pi / 4\right|<\pi / 4+\pi / 200,
$$

with $c_{0}^{ \pm}:=c^{ \pm}\left(J_{0}\right)$. We put

$$
c_{*}:=c_{0}^{+} \nu_{0}+1
$$

where $\nu_{0}$ is a positive integer such that

$$
6 / K<c_{0}^{-} \nu_{0} \sin (\pi / 200)
$$


(ii) In Lemma 2.4, put $J=J_{0}, \zeta_{0}=\varpi\left(\kappa_{b}, 0\right)=u_{0}(0)=v(b)$ implying $\left|\zeta_{0}\right|=K$ (cf. (3.7)). Then, by Lemma 2.4 and the periodicity of $\varpi\left(\kappa_{b}, x\right)$, there exists a curve $\Gamma_{*}\left(\kappa_{b}\right)$ joining 0 to $\nu_{0} \omega\left(\kappa_{b}\right)$ such that the length of it does not exceed $\nu_{0} L_{0}$ and such that

$$
\Lambda_{0,-}<\left|\varpi\left(\kappa_{b}, x\right)\right|<\Lambda_{0,+} \quad \text { along } \Gamma_{*}\left(\kappa_{b}\right)
$$

for $L_{0}:=L\left(J_{0}, K\right), \Lambda_{0,-}:=\Lambda_{-}\left(J_{0}\right)$ and $\Lambda_{0,+}:=\Lambda_{+}\left(J_{0}, K\right)$.

(iii) Put $\rho_{\infty}=2 r_{0}^{2}+\nu_{0} L_{0}+2$. Since $|b|>|\tau|-1>\rho_{\infty}-1$ (cf. (3.7)),

$$
\left\{t|| t-b \mid<\nu_{0} L_{0}+1\right\} \subset\left\{t|| t \mid>2 r_{0}^{2}\right\}
$$

We apply Lemma 2.1 with $\varepsilon=b^{-1}$ to (3.9). In its application, we put $\varphi_{0}(x)=u_{0}(x)=$ $\varpi\left(\kappa_{b}, x\right), \varphi(\varepsilon, x)=u(b, x)$, and choose the curve and the constants as follows:

$\gamma=\Gamma_{*}\left(\kappa_{b}\right), \quad L=\nu_{0} L_{0}, \quad \xi_{0}=\nu_{0} L_{0}+1, \quad R_{0, \pm}=\Lambda_{0, \pm}, \quad \xi_{1}=\left(\nu_{0} L_{0}+1\right)^{-1} / 2$

and

$$
R_{1}=\Lambda_{1}:=2\left(J_{0}+\Lambda_{0,+}^{3}+4 \Lambda_{0,+}^{2}+4 \Lambda_{0,+}\right)^{1 / 2} \Lambda_{0,+}^{1 / 2},
$$

because $\left|\dot{\varpi}\left(\kappa_{b}, x\right)\right|=\left|P\left(\kappa_{b}, \varpi\left(\kappa_{b}, x\right)\right)\right|^{1 / 2}<\Lambda_{1}$ along $\Gamma_{*}\left(\kappa_{b}\right)$ (cf. (3.14)). Note that all the constants above are independent of $\kappa_{b},\left|\kappa_{b}\right| \leqslant J_{0}$, and that all the suppositions of Lemma 2.1 are fulfilled. Then, for $|b|>\varepsilon_{0}^{-1}$, we have

$$
\left|u\left(b, \nu_{0} \omega\left(\kappa_{b}\right)\right)-\varpi\left(\kappa_{b}, \nu_{0} \omega\left(\kappa_{b}\right)\right)\right|=\left|v\left(b+\nu_{0} \omega\left(\kappa_{b}\right)\right)-v(b)\right| \leqslant 1,
$$

where $\varepsilon_{0}=\varepsilon_{0}\left(\nu_{0} L_{0}, \Lambda_{0, \pm}, \Lambda_{1}, 1\right)$ is independent of $\tau$; if necessary, we re-choose $\rho_{\infty}$ so large that $\rho_{\infty}>\varepsilon_{0}^{-1}+1$. By $(3.16), K^{\prime}=\left|v\left(b+\nu_{0} \omega\left(\kappa_{b}\right)\right)\right| \geqslant|v(b)|-1=K-1$. By (3.1), (3.15) and Lemma 3.2 with $K^{\prime}$, we see that the point $b^{\prime}:=b+\nu_{0} \omega\left(\kappa_{b}\right)$ belongs to the domain $|t|>2 r_{0}^{2}$, and that there exists a pole $\tau^{\prime}$ of $v(t)$ satisfying $\left|b^{\prime}-\tau^{\prime}\right|<2 /(K-1)<3 / K$; this, together with (3.7), yields $|b-\tau|+\left|b^{\prime}-\tau^{\prime}\right|<5 / K$. Hence, by (3.11) and (3.13),

$$
\begin{aligned}
\left|\arg \left(\tau^{\prime}-\tau\right)-\pi / 4\right| & =\left|\arg \left(b^{\prime}-b+\left(\tau^{\prime}-b^{\prime}\right)-(\tau-b)\right)-\pi / 4\right| \\
& \leqslant\left|\arg \left(b^{\prime}-b\right)-\pi / 4\right|+\sin ^{-1}\left(\left(\left|\tau^{\prime}-b^{\prime}\right|+|\tau-b|\right) /\left|b^{\prime}-b\right|\right) \\
& \leqslant \pi / 4+\pi / 200+\sin ^{-1}\left((5 / K) /\left(c_{0}^{-} \nu_{0}\right)\right) \\
& <\pi / 4+\pi / 100,
\end{aligned}
$$

and, by (3.12) and (3.13),

$$
\begin{aligned}
& \left|\tau^{\prime}-\tau\right| \leqslant\left|\nu_{0} \omega\left(\kappa_{b}\right)\right|+|b-\tau|+\left|b^{\prime}-\tau^{\prime}\right|<c_{0}^{+} \nu_{0}+5 / K<c_{*}, \\
& \left|\tau^{\prime}-\tau\right| \geqslant\left|b-b^{\prime}\right|-\left|\tau^{\prime}-b^{\prime}\right|-|\tau-b| \geqslant c_{0}^{-} \nu_{0}-5 / K>6 / K-5 / K=1 / K .
\end{aligned}
$$

This completes the proof of Lemma 3.3. 


\subsection{Completion of the proof of Theorem 1.1}

Fix the positive number $\rho_{\infty}$ of Lemma 3.3. Consider the domains

$$
D_{k}:=\left\{t|| \arg \left(t-\rho_{\infty} \mathrm{e}^{(1+2 k) \pi \mathrm{i} / 4}\right)-(1+2 k) \pi / 4 \mid<\pi / 4+\pi / 100\right\} \subset\left\{t|| t \mid>\rho_{\infty}\right\}
$$

$(k=0,1,2,3)$ in the $t$-plane, the union of which covers the full neighbourhood of $t=\infty$. By the transcendency of $w(z)$, there exists a pole $\sigma$ of $w(z)$ such that the corresponding pole $\tau_{0}=\sigma^{2} / 2$ of $v(t)$ belongs to $\bigcup_{k=0}^{3} D_{k}$ (see $[\mathbf{4}, \S 10]$ ); without loss of generality, we may suppose that $\tau_{0} \in D_{0}$ (cf. Remark 3.4). Note that $D_{0} \subset\{t|| \arg t-\pi / 4 \mid<$ $\left.\pi / 4+\pi / 100,|t|>\rho_{\infty}\right\}$. By Lemma 3.3 with $\tau=\tau_{0}$, there exists a pole $\tau_{1}$ satisfying

$$
0<\left|\tau_{1}-\tau_{0}\right|<c_{*}, \quad\left|\arg \left(\tau_{1}-\tau_{0}\right)-\pi / 4\right|<\pi / 4+\pi / 100 .
$$

This implies that $\tau_{1} \in D_{0}$ and that

$$
D_{0}^{(1)}:=\left\{t|| \arg \left(t-\tau_{1}\right)-\pi / 4 \mid<\pi / 4+\pi / 100\right\} \subset D_{0} .
$$

Therefore, Lemma 3.3 is applicable to $\tau=\tau_{1}$ as well, and there exists another pole $\tau_{2}$ satisfying the relations

$$
\tau_{2} \in D_{0}^{(1)} \subset D_{0}
$$

and

$$
D_{0}^{(2)}:=\left\{t|| \arg \left(t-\tau_{2}\right)-\pi / 4 \mid<\pi / 4+\pi / 100\right\} \subset D_{0}^{(1)} \subset D_{0} .
$$

We can repeat this procedure and we get an infinite sequence of poles $\left\{\tau_{\nu}\right\}_{\nu \geqslant 0}$ of $v(t)$ such that

$$
\left|\arg \left(\tau_{\nu+1}-\tau_{\nu}\right)-\pi / 4\right|<\pi / 4+\pi / 100, \quad 0<\left|\tau_{\nu+1}-\tau_{\nu}\right|<c_{*} .
$$

This implies that $n(\rho, v) \geqslant \rho / c_{*}+O(1)$, and hence $n(r, w) \gg n\left(r^{2} / 2, v\right) \gg r^{2}$, which completes the proof of Theorem 1.1 for (IV).

\section{Outline of the proof of Theorem 1.1 for (II)}

Theorem 1.1 for (II) is proved by an argument parallel to that used for (IV).

A. Let $w(z)$ be an arbitrary transcendental solution of (II). Then $v=v(t)=z^{-1 / 2} w(z)$ with $t=(2 / 3) z^{3 / 2}$ is a solution of

$$
v_{t t}=2 v^{3}+v-t^{-1} v_{t}+(2 / 3) \alpha t^{-1}+(1 / 9) t^{-2} v .
$$

The function

$$
V(t):=v_{t}(t)^{2}-v(t)^{4}-v(t)^{2}-(4 / 3) \alpha t^{-1} v(t)-(1 / 9) t^{-2} v(t)^{2}
$$

satisfies

$$
V_{t}+2 t^{-1} V=-2 t^{-1} v(t)^{4}-2 t^{-1} v(t)^{2}-(4 / 3) \alpha t^{-2} v(t)
$$


from which we derive

$$
V(t)=t_{0}^{2} V\left(t_{0}\right) t^{-2}-2 t^{-2} \int_{C\left(t_{0}, t\right)}\left(s v(s)^{4}+s v(s)^{2}+(2 / 3) \alpha v(s)\right) \mathrm{d} s,
$$

where $C\left(t_{0}, t\right)$ is a path joining $t_{0}$ to $t$. The corresponding elliptic function $\varpi_{*}(\kappa, x)$, $\kappa \in \mathbb{C}$, satisfies

$$
\dot{u}^{2}=\hat{P}(\kappa, u)=u^{4}+u^{2}+\kappa .
$$

For each $q \in \mathbb{Z}$, and for each $\kappa,|\kappa| \leqslant J$, we find a period $\omega(\kappa)$ of $\varpi_{*}(\kappa, x)$ with the same property as in Lemma 2.3. Furthermore, if $|\kappa| \leqslant J$, and if $\varpi_{*}(\kappa, 0)=\zeta_{0},\left|\zeta_{0}\right|>10$, then there exists a curve $\Gamma_{\kappa}\left(\zeta_{0}\right)$ joining 0 to $\omega(\kappa)$ with the property (i) of Lemma 2.4 and

(ii') $\left|\varpi_{*}(\kappa, x)\right|<\Lambda\left(J,\left|\zeta_{0}\right|\right)$ along $\Gamma_{\kappa}\left(\zeta_{0}\right)$ for some $\Lambda\left(J,\left|\zeta_{0}\right|\right)$ independent of $\kappa$.

B. Instead of (2.10), consider the auxiliary function

$$
\Psi(z):=w^{\prime}(z)^{2}-w(z)^{4}-z w(z)^{2}-2 \alpha w(z) .
$$

Take a circle $|z|=r_{0}>10$ on which $w(z) \neq 0, \infty$. For $K$ satisfying $K>2 r_{0}^{-1 / 2} M\left(w ; r_{0}\right)+$ $M\left(\Psi ; r_{0}\right)+10^{2}+2|\alpha|$, we put $D_{K}:=\left\{\left.z|| w(z)|>K| z\right|^{1 / 2}\right\}$. Starting from [12, Proposition 5.1], by the same argument as in $\S 2.3$, we obtain the (II) versions of Lemmas 2.8 and 2.9 .

Lemma 4.1. Suppose that $a,|a|>r_{0}$, satisfies $|w(a)|=K|a|^{1 / 2}$. Then there exist a pole $\sigma_{a}^{\sharp}$ of $w(z)$ and the disc

$$
\hat{\Delta}\left(\sigma_{a}^{\sharp}\right):\left|z-\sigma_{a}^{\sharp}\right|<(11 / 10) K^{-1}\left|\sigma_{a}^{\sharp}\right|^{-1 / 2}
$$

such that $a \in \hat{\Delta}\left(\sigma_{a}^{\sharp}\right)$.

Lemma 4.2. Let $\sigma$ be an arbitrary pole of $w(z)$ such that $|\sigma|>2 r_{0}$. Then, there exist a point $a^{\sigma} \in \hat{\Delta}(\sigma)$ and a curve $\hat{C}_{0}\left(a^{\sigma}\right) \subset\left\{z|| z \mid \geqslant r_{0}\right\}$ with the following properties:

(i) $\hat{C}_{0}\left(a^{\sigma}\right)$ joins a point $z^{\sigma},\left|z^{\sigma}\right|=r_{0}$, to $a^{\sigma}$, and the length of it does not exceed $\pi\left|a^{\sigma}\right| / 2$; and

(ii) $|w(z)| \leqslant K|z|^{1 / 2}$ along $\hat{C}_{0}\left(a^{\sigma}\right)$, and $\left|w\left(a^{\sigma}\right)\right|=K\left|a^{\sigma}\right|^{1 / 2}$.

C. Make the replacement $K \rightarrow K-1$ in the supposition on $K$ (cf. (3.1)). Concerning $v(t)$, instead of Lemmas 3.1 and 3.2, we have the following.

Lemma 4.3. Let $\sigma$ be an arbitrary pole of $w(z)$ such that $|\sigma|>2 r_{0}$, and let $a^{\sigma}$ be the point given by Lemma 4.2. Then the corresponding point $b^{\sigma}=(2 / 3)\left(a^{\sigma}\right)^{3 / 2}$ satisfies $\left|b^{\sigma}-(2 / 3) \sigma^{3 / 2}\right|<2 / K,\left|v\left(b^{\sigma}\right)\right|=K,\left|V\left(b^{\sigma}\right)\right| \leqslant J_{0}$, and $\left|v_{t}\left(b^{\sigma}\right)^{2}-v\left(b^{\sigma}\right)^{4}-v\left(b^{\sigma}\right)^{2}\right| \leqslant J_{0}$ for some $J_{0}=J_{0}(K)$ independent of $\sigma$.

Lemma 4.4. Suppose that $b,|b|>r_{0}^{3 / 2}$, satisfies $|v(b)|=K$. Then there exists a pole $\tau_{b}$ of $v(t)$ such that $\left|b-\tau_{b}\right|<2 / K$. 
D. The union of the sectors $\hat{S}_{q}:|\arg z-(2 q-1) \pi / 6|<\pi / 6+\delta(-2 \leqslant q \leqslant 3)$ covers the full neighbourhood of $z=\infty$. The image of $\hat{S}_{q}$ under the mapping $t=(2 / 3) z^{3 / 2}$ is $\hat{T}_{q}:|\arg t-(2 q-1) \pi / 4|<\pi / 4+3 \delta / 2$. For an arbitrary pole $\tau \in \hat{T}_{1}$ (with $3 \delta / 2=\pi / 100$ ) of $v(t)$ with sufficiently large modulus, we can find another pole $\tau^{\prime}$ with the same property as (3.6). To show this fact, in place of (3.9), we treat the perturbed equation

$$
\ddot{u}=2 u^{3}+u-\frac{b^{-1}(\dot{u}-2 \alpha / 3)}{1+b^{-1} x}+\frac{b^{-2} u}{9\left(1+b^{-1} x\right)^{2}}
$$

for $b$ close to $\tau$. This is obtained from (4.1) by the change of variables $t=b+x$, $u(b, x)=v(b+x)$. Let $\varpi_{*}\left(\kappa_{b}, x\right)$ be a solution of $(4.2)$ with $\kappa=\kappa_{b}:=v_{t}(b)^{2}-v(b)^{4}-$ $v(b)^{2}$ satisfying $\varpi_{*}\left(\kappa_{b}, 0\right)=v(b), \dot{\varpi}_{*}\left(\kappa_{b}, 0\right)=v_{t}(b)$. The solution of (4.3) with the initial condition $u(b, 0)=v(b), \dot{u}(b, 0)=v_{t}(b)$ is asymptotic to $\varpi_{*}\left(\kappa_{b}, x\right)$ in a suitable domain. By the same argument as used in $\S 3.3$ together with Remark 2.2 , we construct a sequence of poles of $v(t)$ satisfying $n(\rho, v) \gg \rho$, which implies the estimate $n(r, w) \gg r^{3 / 2}$.

Acknowledgements. The author is grateful to the referee for an important and helpful comment concerning a crucial part of the proof (in §3.3) of the main result, and also to Professor Nalini Joshi for a useful discussion on Boutroux asymptotics.

\section{References}

1. P. Boutroux, Recherches sur les transcendents de M. Painlevé et l'étude asymptotique des équations différentielles du second ordre, Annls Sci. Ec. Norm. Super. 30 (1913), $255-375$.

2. P. Boutroux, Recherches sur les transcendents de M. Painlevé et l'étude asymptotique des équations différentielles du second ordre (suite), Annls Sci. Ec. Norm. Super. 31 (1914), 99-159.

3. E. A. CodDington AND N. Levinson, Theory of ordinary differential equations (McGraw-Hill, New York, 1955).

4. V. I. Gromak, I. Laine and S. Shimomura, Painlevé differential equations in the complex plane (Walter de Gruyter, Berlin, New York, 2002).

5. W. K. Hayman, Meromorphic functions (Clarendon, Oxford, 1964).

6. A. HinkKanen And I. Laine, Solutions of the first and second Painlevé equations are meromorphic, J. Analyse Math. 79 (1999), 345-377.

7. A. Hinkkanen And I. Laine, Growth results for Painlevé transcendents, Math. Proc. Camb. Phil. Soc., in press.

8. N. Joshi, Asymptotic studies of the Painlevé equations, The Painlevé property, one century later (ed. R. Conte), pp. 181-227 (Springer, 1999).

9. I. LAINE, Nevanlinna theory and complex differential equations (Walter de Gruyter, Berlin, New York, 1993).

10. E. Mues AND R. Redheffer, On the growth of the logarithmic derivatives, J. Lond. Math. Soc. 8 (1974), 412-425.

11. K. Окамото And K. Takano, The proof of the Painlevé property by Masuo Hukuhara, Funkcial. Ekvac. 44 (2001), 201-217.

12. S. ShimomurA, Growth of the first, the second and the fourth Painlevé transcendents, Math. Proc. Camb. Phil. Soc. 134 (2003), 259-269.

13. S. Shimomura, Lower estimates for the growth of Painlevé transcendents, Funkcial. Ekvac. 46 (2003), 287-295. 
14. S. Shimomura, Proofs of the Painlevé property for all Painlevé equations, Jpn. J. Math. 29 (2003), 159-180.

15. N. Steinmetz, On Painlevé's equations I, II and IV, J. Analyse Math. 82 (2000), 363-377.

16. N. Steinmetz, Value distribution of the Painlevé transcendents, Isr. J. Math. 128 (2002), $29-52$.

17. N. Steinmetz, Global properties of the Painlevé transcendents, submitted.

18. N. Steinmetz, Boutroux's method vs. re-scaling, Lower estimates for the orders of growth of the second and fourth Painlevé transcendents, submitted.

19. K. TAKAnO, Defining manifolds for Painlevé equations, Toward the exact WKB analysis of differential equations, linear or nonlinear (ed. C. J. Howls, T. Kawai and Y. Takei), pp. 261-269 (Kyoto University Press, Kyoto, 2000). 